Check for updates

Cite this: RSC Adv., 2017, 7, 34125

\title{
A superhydrophobic solar selective absorber used in a flat plate solar collector $\dagger$
}

\author{
Yanqing Zhu, (D) ab Jifu Shi, ${ }^{\text {ad }}$ Qizhang Huang, ${ }^{\text {ab }}$ Yueyun Fang, ${ }^{\text {ab }}$ Leilei Wang $^{\text {a }}$ \\ and Gang $X^{* a c}$
}

A new kind of superhydrophobic (SH) solar selective absorber (SSA) used in a low-temperature flat plate solar collector is proposed. Getting rid of the glass cover can improve the performance of the flat plate solar collector. SSA with a SH coating can maintain the efficiency in a practical outdoor working environment. The SH coating can be made by a simple Sol-Gel method; the water contact angle of $\mathrm{SH}$ SSA can reach up to $157^{\circ}$ and the sliding angle is less than $2^{\circ}$. The self-cleaning of SH SSA can be realized by rainwater or natural wind. The solar absorptance of SH SSA is still $89.46 \%$ after treating in a neutral salt spray for $48 \mathrm{~h}$, which indicates that SH SSA has good corrosion resistance and is suitable to the outdoor environment. Thermal performance tests show that the flat plat solar collector with SH SSA has excellent thermal performance for low-temperature application.

Received 14th April 2017 Accepted 3rd July 2017

DOI: $10.1039 / \mathrm{c} 7 \mathrm{ra0} 4238 \mathrm{~h}$

rsc.li/rsc-advances working environmental condition is rather dirty, and the accumulations of surrounding dust on the glass cover reduce the transmittance of normally incident light, which can largely decrease the performance of the solar thermal system.

To alleviate reflection loss on the air/glass interface and improve the efficiency of the incident light, many efforts have been focused on antireflective coatings on the glass cover. The glass cover coated with silica, ${ }^{12}$ double-layered $\mathrm{SiO}_{2} / \mathrm{TiO}_{2}$ (ref. 13) or $\mathrm{SiO}_{2} / \mathrm{TiO}_{2} / \mathrm{SiO}_{2}-\mathrm{TiO}_{2}$ (ref. 14) multilayer antireflective films can increase the transmittance of incident light to some extent, but the problem of dust accumulations has not been solved. The dust accumulated on the surfaces of solar thermal system has been commonly cleaned by artificial cleaning to maintain optimum performance, which is time-consuming and manpower-wasting. Therefore, self-cleaning coating is an effective solution to increase the efficiency of the solar thermal system. Superhydrophobic (SH) self-cleaning surface which is known as "Lotus effect" has been studied. ${ }^{15,16}$ SH surface with water contact angle (WCA) greater than $150^{\circ}$ and sliding angle less than $10^{\circ}$ is usually caused by a rough surface with hierarchical micro- and nanostructures and formed by a material with low surface energy. ${ }^{17-19}$ There are a number of methods for obtaining SH surface such as Sol-Gel method, ${ }^{20,21}$ layer-by-layer assembly, ${ }^{22}$ reactive ion etching ${ }^{23}$ and spray deposition. ${ }^{24-26}$ Among them, Sol-Gel method is a popular method for preparation of inorganic materials and has the advantages of lower processing temperature, high homogeneity and purity of resulting materials and possibility of various forming processes. $^{27}$

It is well known that glass cover can prevent heat convection loss and play a role of heat preservation to obtain high temperature water in the flat plate solar collector. However, the Guangdong, China

$\dagger$ Electronic supplementary information (ESI) available. See DOI: 10.1039/c7ra04238h 
losses caused by reflection and absorption of the glass cover may be more serious than heat convection loss in lowtemperature applications. Then the SSA which has good corrosion resistance and self-cleaning property can be directly exposure to the outside environment. The flat plate solar collectors using this SSA without glass cover can solve the problems of incident light loss and dust accumulations and also have good weather resistance.

In this paper, we reported for the first time a new kind of $\mathrm{SH}$ SSA used in the low-temperature flat plate solar collector. The SH coating is directly coated on the surface of SSA to form a selfcleaning surface. The fabricated SH SSA has a contact angle of $157^{\circ}$ and sliding angle of less than $2^{\circ}$. The SH SSA exhibits high self-cleaning property via water droplet or wind. The SH SSA has good corrosion resistance and can be suitable to the outdoor environment. In addition, thermal performance test of flat plate solar collectors with SH SSA has been made, and the results indicate that it has more excellent thermal performance at lowtemperature application.

\section{Experimental sections}

\subsection{Fabrication of $\mathrm{SH}$ coating}

To obtain the $\mathrm{SH}$ coating, the $\mathrm{SiO}_{2}$ nanospheres have been used to build rough structures and $1 H, 1 H, 2 H, 2 H$-perfluorooctyltriethoxysilane has been used as low surface energy material. It is well known that the superhydrophobic performance has been affected by the sizes and morphologies of the $\mathrm{SiO}_{2}$ nanospheres dramatically. Therefore, the $\mathrm{SiO}_{2}$ collosol with sizes of 80, 100, 150 and $300 \mathrm{~nm}$ were made and coated with dip-coating method. The different morphologies have been present and the wettabilities of surfaces have been measured. The coatings with 80 and $100 \mathrm{~nm} \mathrm{SiO}$ nanospheres have well $\mathrm{SH}$ properties with water contact angles of $156^{\circ}$ and $157^{\circ}$ respectively (see Fig. S3†). The detail fabrication processes and corresponding test results can be seen in ESI. $\dagger$ Here, we only describe the fabrication process for the coating with mean size of $100 \mathrm{~nm} \mathrm{SiO}{ }_{2}$ nanospheres which has good SH property.

$18.4 \mathrm{ml}$ of tetraethoxysilane (TEOS) was added into $300 \mathrm{ml}$ of absolute ethanol, and $32.4 \mathrm{ml}$ of ammonia solution $\left(\mathrm{NH}_{4} \mathrm{OH}, 30\right.$ $\mathrm{wt} \%$ ) was added into $100 \mathrm{ml}$ of absolute ethanol, followed by ultrasonic oscillation for 10 min to obtain two kinds of even solutions. Mixed the two kinds of solutions into a sealed flask and stirred in $50{ }^{\circ} \mathrm{C}$ constant temperature water bath for about $1 \mathrm{~h}$ to form $\mathrm{SiO}_{2}$ collosol. Then the $\mathrm{SiO}_{2}$ collosol was poured into a beaker and stirred in opening state at the room temperature until the ammonia was exhausted. $1 \mathrm{H}, 1 \mathrm{H}, 2 \mathrm{H}, 2 \mathrm{H}$-Perfluorooctyltriethoxysilane which has low surface energy was added into the $\mathrm{SiO}_{2}$ collosol with a mass fraction of $1 \%$ and stirred for about $2 \mathrm{~h}$ in sealed state at the room temperature, then the $\mathrm{SH} \mathrm{SiO}_{2}$ collosol was obtained. Finally, the $\mathrm{SH} \mathrm{SiO}_{2}$ collosol was coated on the SSA by dip-coating, spraying or spincoating and dried in air for at least $2 \mathrm{~min}$. Then the self-cleaning SSA was obtained.

The coatings have been formed by dip-coating, spraying or spin-coating method, and different morphologies could be present (see ESI, Fig. S4†). Coating formed by dip-coating method has relatively rough structure made by random distributions of nanospheres. Coating formed by spraying method is relatively thick. Coating formed by spin-coating method produces uneven thickness in the middle and edge. In the paper, the coating was obtained by dip-coating method using self-developed vertical lift coating machine. The soaking time was $1 \mathrm{~min}$, the pulling speed was $2 \mathrm{~cm} \mathrm{~min}^{-1}$, and the drying time was about 2 min after pulling. In addition, the SSA used in the paper with stainless steel as substrate and $\mathrm{Si}_{3} \mathrm{~N}_{4}$ as surface layer was fabricated by our team. ${ }^{28,29}$

\subsection{Salt spray test}

A salt fog box was used to determinate the resistance to neutral salt spray. The temperature of the salt fog box was $35{ }^{\circ} \mathrm{C}$, and salt $(\mathrm{NaCl})$ concentration was $50 \mathrm{~g} \mathrm{l}^{-1}, \mathrm{pH}$ 6.5-7.2. The volume of spray was $2 \mathrm{ml} \mathrm{h}^{-1}$ in $80 \mathrm{~cm}^{2}$.

\subsection{Thermal performance test of flat plate solar collector}

Flat plate solar collectors whose light area is $0.03 \mathrm{~m}^{2}$ of SH SSA without glass cover and SSA with glass cover have been made. The thermal performance tests were tested using a solar simulator (Abet, SUN 3000) under solar insolation of $1000 \mathrm{~W} \mathrm{~m}^{-2}$. The water temperatures of the flat plate solar collector and the ambient temperature are measured by a multi-channel temperature acquisition system.

\subsection{Characterization}

A Hitachi S-4800 scanning electron microscope (SEM) was used to acquire surface images at an accelerating voltage of $2 \mathrm{kV}$. The water contact angles are measured at ambient temperature via the sessile-drop method using an optical contact angle meter (Dataphysics OCA 20LHT, water droplet is $5 \mu \mathrm{l}$ ). UV-Vis-NIR spectra were measured on a Perkin Elmer Lambda 750 spectrophotometer with an integrating sphere in spectral region of 300-2500 nm using $\mathrm{BaSO}_{4}$ as a reference. The infrared reflectance spectra were obtained on a Bruker Tensor 27 spectrophotometer in the range of 2500-25 $000 \mathrm{~nm}$ with an integrating sphere. In the paper, the test of infrared reflectance spectra which is subject to environmental impact has some certain deviation, but it doesn't affect the final contrast results.

The solar absorptance and thermal emittance can be calculated through reflectance measurement $R(\lambda)$ as follow:

$$
\begin{gathered}
\alpha=\frac{\int_{300 \mathrm{~nm}}^{2500 \mathrm{~nm}}[1-R(\lambda)] P_{\text {sun }}(\lambda) \mathrm{d} \lambda}{\int_{300 \mathrm{~nm}}^{2500 \mathrm{~nm}} P_{\text {sun }}(\lambda) \mathrm{d} \lambda} \\
\varepsilon=\frac{\int_{2500 \mathrm{~nm}}^{25000 \mathrm{~nm}}[1-R(\lambda)] P_{\mathrm{B}}(\lambda) \mathrm{d} \lambda}{\int_{2500 \mathrm{~nm}}^{25000 \mathrm{~nm}} P_{\mathrm{B}}(\lambda) \mathrm{d} \lambda}
\end{gathered}
$$

where, $P_{\operatorname{sun}}(\lambda)$ is the normal solar spectral irradiance defined by the ISO standard 9845-1-(1992) for air mass (AM) 1.5. $P_{\mathrm{B}}(\lambda)$ is given by Plank's law, 


$$
P_{\mathrm{B}}=\frac{C_{1}}{\lambda^{5}\left(\mathrm{e}^{C_{2} / \lambda T}-1\right)}
$$

here, $C_{1}=3.743 \times 10^{-16} \mathrm{~W} \mathrm{~m}^{2}, C_{2}=1.4387 \times 10^{-2} \mathrm{~m} \mathrm{~K}, T=$ $25{ }^{\circ} \mathrm{C}$.

\section{Results and discussion}

SH SSA is SSA coated with $\mathrm{SH}$ coating. The $\mathrm{SH}$ coating was fabricated by simple Sol-Gel method. The $\mathrm{SH} \mathrm{SiO}_{2}$ collosol was made with the TEOS as the precursor, ammonia solution as catalyst and $1 H, 1 H, 2 H, 2 H$-perfluorooctyltriethoxysilane as low surface energy material. Then the SSA was coated with $\mathrm{SH} \mathrm{SiO}_{2}$ collosol by dip-coating, spraying or spin-coating to obtain $\mathrm{SH}$ SSA.

The photographs of SSA before and after coated with $\mathrm{SH}$ coating are shown in Fig. 1(a) and (b), respectively. After coated with SH coating, there is no other obvious difference except slight change in color. The surface morphologies of SSA before and after coated with SH coating measured using scanning electron microscope (SEM) images are shown in Fig. 1(c) and (d), respectively. Small irregular particles and relatively flat surface can be seen on the surface of SSA in Fig. 1(c). After coated with $\mathrm{SH}$ coating, $\mathrm{SiO}_{2}$ nanospheres with the mean size about $100 \mathrm{~nm}$ can be seen on the surface of SH SSA in Fig. 1(d). Some of the nanospheres are distributed in a single layer and some are randomly overlapped. The random distribution of nanospheres makes the surface of SSA relatively rough, which leads to the $\mathrm{SH}$ surface. For the problem of poor adhesion
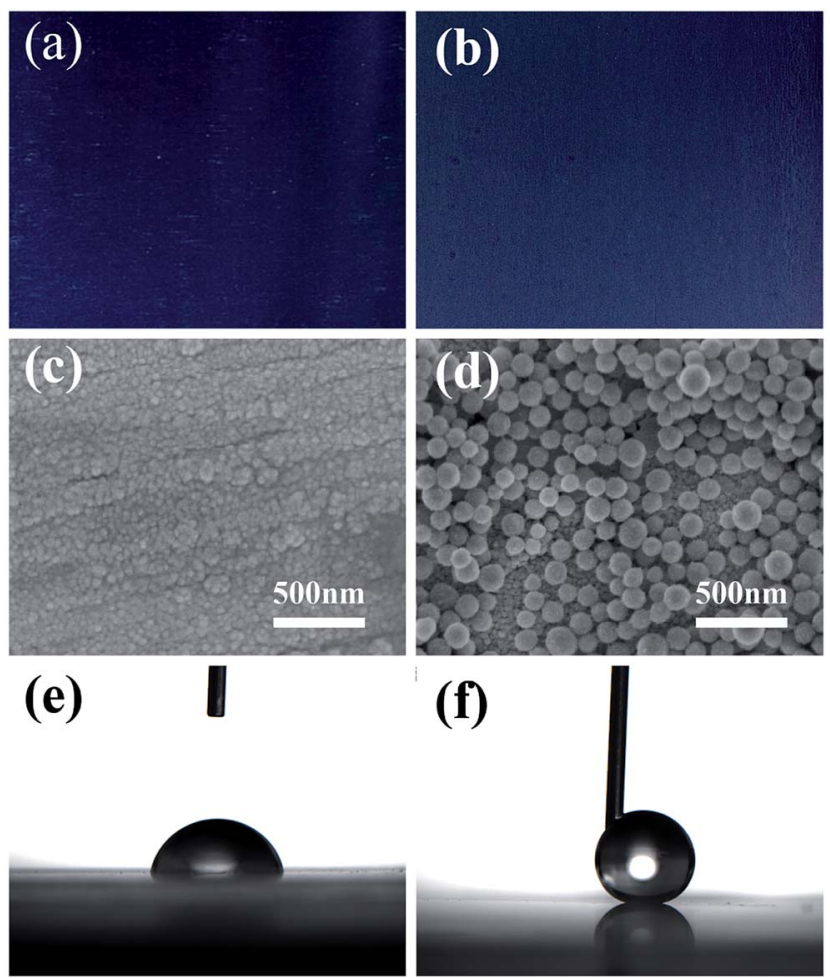

Fig. 1 Photographs, SEM images and WCAs of $(a, c, e)$ SSA and $(b, d, f)$ SH SSA. between the SH coating and SSA, we have proposed s sandwich structure of "substrate + adhesive + coating" which can provide a reference to solve the problem. ${ }^{21}$ The SSA was coated with organosilicon and superhydrophobic coating sequentially and samples with sandwich structure were obtained (see Fig. S5 $\dagger$ ). The mechanical property tests of the samples have been made. The results show that the water contact angles of the samples can still be $150^{\circ}$ after five times of tape peeling tests and sandpaper abrasion tests respectively (see ESI, Fig. S6†). In the paper, in order to verify the feasibility of using $\mathrm{SH}$ coating in flat plate solar collector, we just consider the situation of SH coating directly used on the surface of SSA.

To investigate the surface wetting properties of each SSA, the WCA measurements with volume of $5 \mu$ l water droplet were carried out. The WCAs of SSA before and after coated with SH coating are shown in Fig. 1(e) and (f), respectively. The WCA of SSA before coated with $\mathrm{SH}$ coating is $75^{\circ}$ mainly because of the relatively flat surface. After coated with $\mathrm{SH}$ coating, the WCA of SH SSA becomes $157^{\circ}$ and sliding angle is less than $2^{\circ}$, which indicates the surface is $\mathrm{SH}$. There are two reasons for the $\mathrm{SH}$ coating. One is the rough surface structure composed of nanospheres and the other is the low surface energy supplied by $1 H, 1 H, 2 H, 2 H$-perfluorooctyltriethoxysilane.

To characterize absorptance and emission property of the SSA before and after coated with $\mathrm{SH}$ coating, the diffuse reflection was measured in spectral region of 300-2500 nm and 2500-25 $000 \mathrm{~nm}$. The reflectance spectra of SSA, SH SSA and SSA covered with glass are shown in Fig. 2. Compared with the
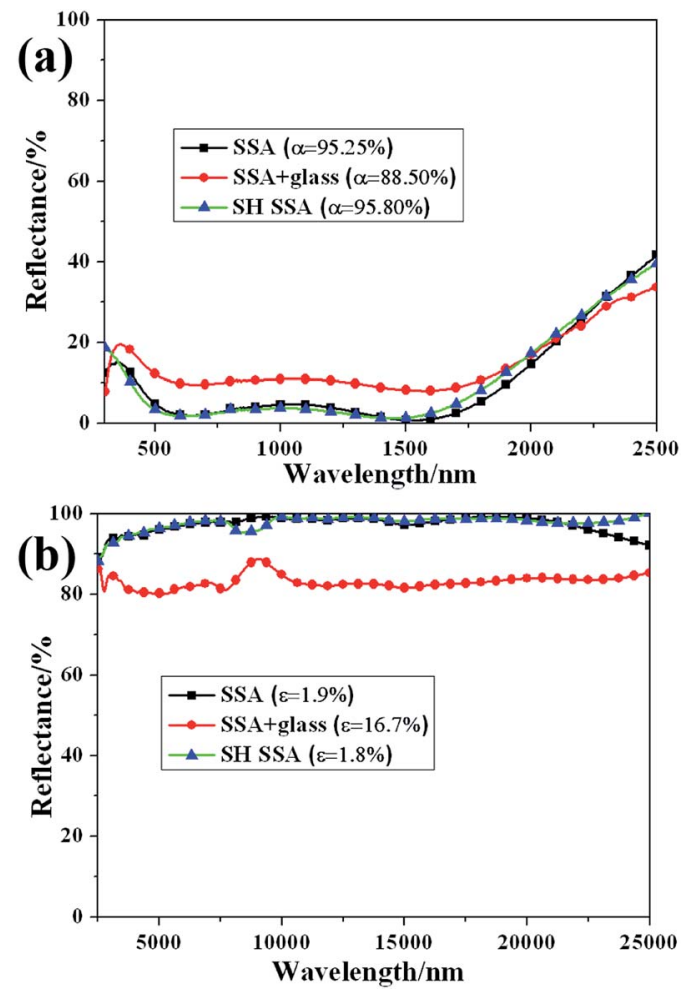

Fig. 2 Diffuse reflectance spectra of SSA, SH SSA, and SSA with borosilicate glass cover above at the wavelength of (a) 300-2500 nm and (b) 2500-25 $000 \mathrm{~nm}$. 
absorptance of SSA before coated with SH coating ( $\alpha=95.25 \%$ ), the absorptance of SSA with borosilicate glass cover above is significantly reduced to $88.50 \%$. Meanwhile, after covered with borosilicate glass above, the thermal emittance is increased from $1.9 \%$ to $16.7 \%$. The reducing of absorptance and increasing of thermal emittance owns to the introduction of borosilicate glass cover. On one hand, the reflection of normally incident light is happened on each air/glass interface. On the other hand, the incident light is partly absorbed by borosilicate glass itself. After coated with SH coating, the values of absorption and thermal emittance of SSA become $95.80 \%$ and $1.8 \%$ respectively, denoting that the thermal performance of SSA are nearly unchanged and even a little better. This result is much better than that of SSA with borosilicate glass cover. Therefore, getting rid of glass cover and coating SSA with SH coating are effective methods to improve thermal efficiency of SSA thermal module.

In order to further explain the function of SH coating, the total transmittance of borosilicate glass uncoated and coated with $\mathrm{SH}$ coating has been made and measured in spectral region of 300-2500 $\mathrm{nm}$ as shown in Fig. 3. From the Fig. 3, it can be seen that the mean total transmittance of borosilicate glass coated with $\mathrm{SH}$ coating increase from $87 \%$ to $89 \%$, which is $2 \%$ higher than the value of borosilicate glass. That is because of the medium refractive index of the SH layer and some diffuse light captured by rough structure. The inset paragraph of the Fig. 3 is the photo of borosilicate glass with partly coated with $\mathrm{SH}$ coating, it can be seen that the letters below the borosilicated glass can be seen clearly, there is no big difference of the letters between the borosilicated glass uncoated and coated with $\mathrm{SH}$ coating. The good transmittance shows that the incident light has not reduced after through SH coating. This result can also explain why the absorptance and thermal emittance of SSA are nearly unchanged after coated with SH coating. In addition, the ball like water droplet indicates good $\mathrm{SH}$ character of the borosilicated glass coated with $\mathrm{SH}$ coating.

The SH SSA had good self-cleaning property, and it can realize self-cleaning by rainwater or natural wind. The selfcleaning processes by water droplet and wind are shown in Fig. 4. The SH SSA was placed at a tilt angle in a Petri dish and

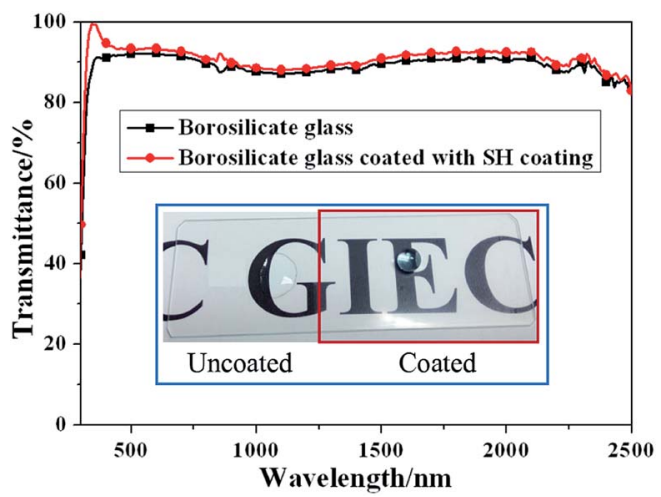

Fig. 3 Total transmittance spectra of borosilicate glass and borosilicate glass coated with $\mathrm{SH}$ coating (inset: photo of borosilicate glass with partly coated with $\mathrm{SH}$ coating).

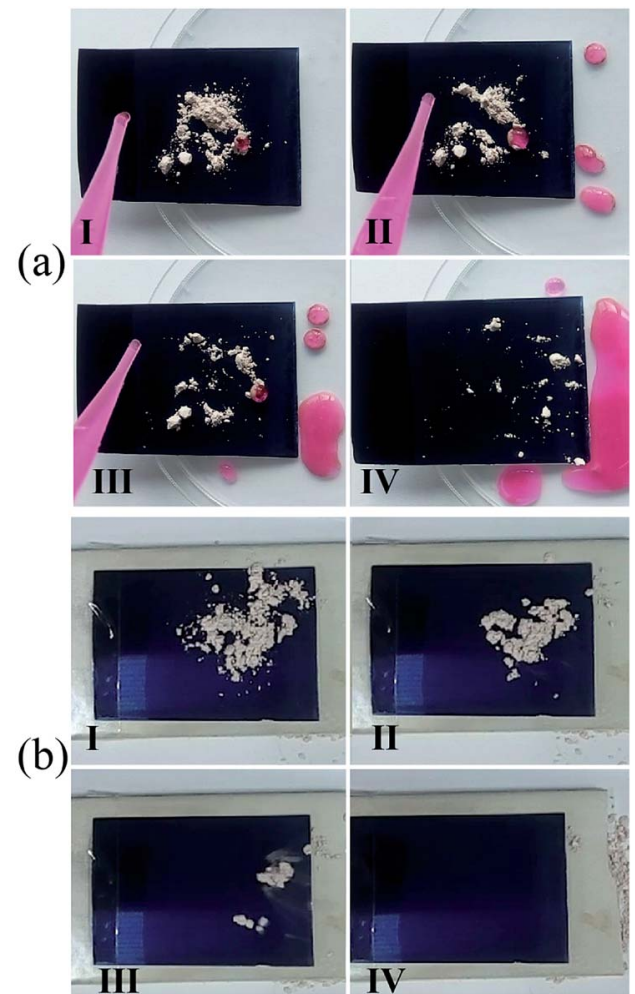

Fig. 4 Self-cleaning test of SH SSA (a) via water droplet and (b) via wind. The dust (diatomite powder) can be easily removed from the $\mathrm{SH}$ SSA via water droplet or wind (I-IV).

artificial dust (diatomite powder) was sprinkled on the surface of SH SSA. Then rhodamine B-dyed water droplets were dropped on the contaminant surface. The dust was immediately picked up and carried away by water droplets during the sliding process, which could make the surface clean (Fig. 4(a), Movie $\mathrm{S} 1 \dagger)$. Meanwhile, the self-cleaning via wind test has been made on the surface of horizontal placed SH SSA. The dust on the surface of SH SSA could be easily taken away completely by a tiny wind with a speed about $3.9 \mathrm{~m} \mathrm{~s}^{-1}$ (Fig. 4(b), Movie S2 $\dagger$ ). Due to the rough surface structure and low surface energy of $\mathrm{SH}$ SSA, the adhesion force between dust and SH SSA is very small, which can easily make the SH SSA clean via water droplet or tiny wind.

In order to verify the corrosion resistance of SH SSA in the outdoor environment, salt spray tests have been made. The reflectance spectra of SSA and SH SSA before and after treating in neutral salt spray $48 \mathrm{~h}$ at the wavelength of 300-2500 $\mathrm{nm}$ and 2500-25 $000 \mathrm{~nm}$ are shown in Fig. 5. It can be found that the solar absorptance of SSA decreases from $95.25 \%$ to $80.97 \%$, and the thermal emittance of SSA increases from $1.9 \%$ to $13.1 \%$. Meanwhile, the solar absorptance of SH SSA decreases from $95.70 \%$ to $89.46 \%$, the thermal emittance of SSA increases from $2.1 \%$ to $12 \%$. These illustrate the surface or the stainless steel substrate may be slightly corroded. However, the corrosion resistance of SH SSA which coated with $\mathrm{SH}$ coating is better than that of SSA. Due to the rough surface structure and low surface energy, the SH coating of SH SSA prevents the salt spray into the 

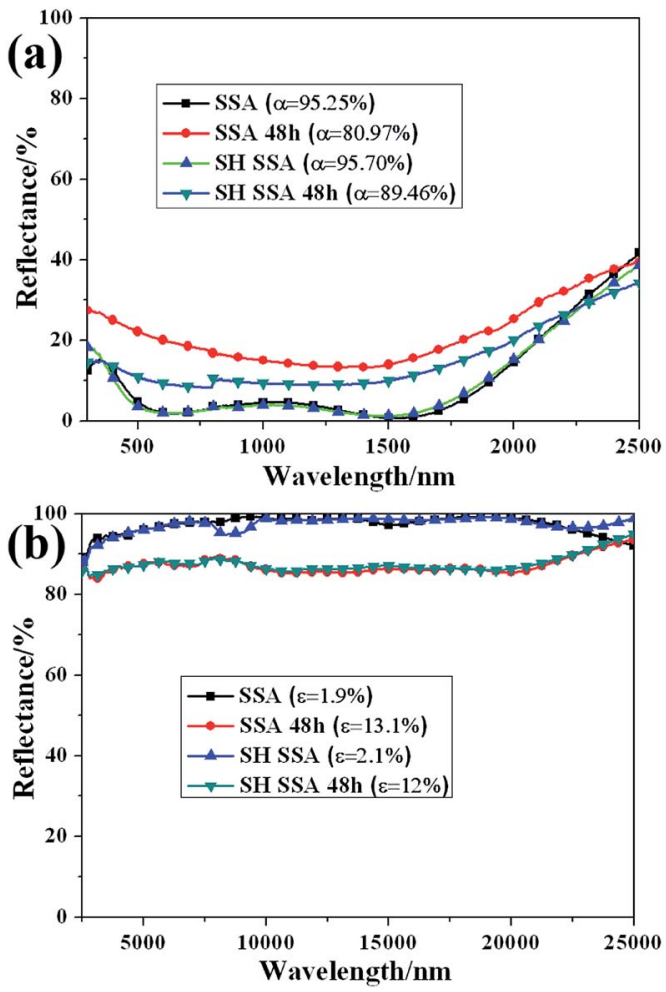

Fig. 5 Reflectance spectra of SSA and SH SSA before and after treating in neutral salt spray for $48 \mathrm{~h}$ at the wavelength of (a) 300-2500 nm and (b) 2500-25 $000 \mathrm{~nm}$.

SSA and protect the coating and the stainless steel substrate corrosion. Therefore, the SH SSA which has good corrosion resistance and can be suitable to the outdoor environment.

To test the SH SSA's practical thermal performance, the SH SSA is applied on a small flat plate solar collector without glass cover above. The contrast test of flat plate solar collectors between SH SSA without glass cover and SSA with glass cover has been made under uniform insolation of $1000 \mathrm{~W} \mathrm{~m}^{-2}$ and ambient temperature of $20{ }^{\circ} \mathrm{C}$. The test results are shown in Fig. 6. It can be seen that the temperatures of flat plate solar collector increase with the exposure time and finally tend to be stable. The stable temperatures of SH SSA without glass and SSA with glass cover are $85{ }^{\circ} \mathrm{C}$ and $88{ }^{\circ} \mathrm{C}$, respectively. However, the temperature increase rate of SH SSA without glass cover is faster

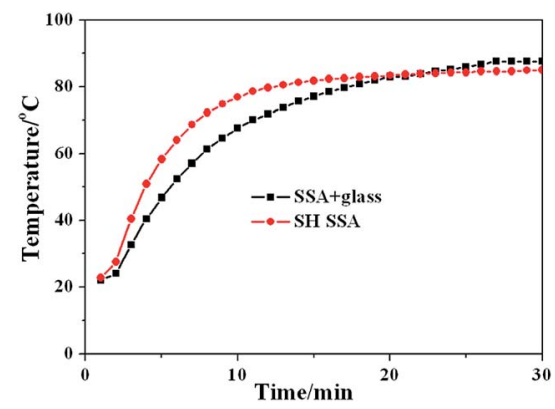

Fig. 6 Flat plate solar collectors' test temperature curves of SSA with glass cover and SH SSA without glass cover. than SSA with glass cover at the beginning, and the temperatures are equal at $83.8{ }^{\circ} \mathrm{C}$. That is to say, the flat plate solar collector with SH SSA without glass cover has more excellent thermal performance below $83.8{ }^{\circ} \mathrm{C}$. Therefore, the flat plate solar collector with SH SSA without glass cover can be more suitable to low-temperature application such as hot water supply for our daily life, solar drying applications, passive solar building.

\section{Conclusions}

In summary, a new kind of SH SSA without glass cover which used in the low-temperature flat plate solar collector has been proposed. Getting rid of glass cover and realizing self-cleaning of SH SSA can improve the efficiency of the solar thermal system. The SH coating can be made by simple Sol-Gel method, the WCA of SH SSA can reach to $157^{\circ}$ and sliding angle is less than $2^{\circ}$. After coated with $\mathrm{SH}$ coating, the absorptance and thermal emittance of SSA are nearly unchanged, which is much better than that of SSA with borosilicate glass cover. The selfcleaning property of SH SSA can be easily realized by water droplet and tiny wind. In addition, the neutral salt spray test has been made, and the solar absorptance of SH SSA is still 89.46\%, which indicates that SH SSA has good corrosion resistance and can be suitable to the outdoor environment. Finally, thermal performance test of flat plate solar collectors shows that the flat plat solar collector with SH SSA without glass cover has more excellent thermal performance below $83.8^{\circ} \mathrm{C}$ and can be more suitable to low temperature application.

\section{Acknowledgements}

This work is supported by Guangdong-Hong Kong Co-operation Project (2014B050505015), Science and Technology Program of Guangdong, China (2014A010106018, 2013A011401011), Construction project of Guangdong solar thermal and solar thermal Advanced Materials Engineering Technology Research Center (2014B090904071), National Natural Science Foundation of China (21103194), Guangdong Special Support Program for High-level Talents (2014TQ01N610), Project of Youth Innovation Promotion Association and Project of Science and Technology Service Network initiative, Chinese Academy of Sciences.

\section{Notes and references}

1 J. Pekez, M. Lambic and S. Stojadinovic, Metal. Int., 2013, 18, 77-80.

2 A. Amri, Z. T. Jiang, T. Pryor, C.-Y. Yin and S. Djordjevic, Renewable Sustainable Energy Rev., 2014, 36, 316-328.

3 K. M. Pandey and R. Chaurasiya, Renewable Sustainable Energy Rev., 2017, 67, 641-650.

4 D. Xiong, W. Chen and L. I. Hong, J. Eng. Sci. Technol. Rev., 2014, 32, 50-58.

5 X.-H. Gao, Z.-M. Guo, Q.-F. Geng, P.-J. Ma, A.-Q. Wang and G. Liu, $R S C$ Adv., 2016, 6, 63867-63873.

6 P. Ma, Q. Geng, X. Gao, S. Yang and G. Liu, RSC Adv., 2016, 6, 54820-54829. 
7 M. Khoukhi and S. Maruyama, Sol. Energy, 2006, 80, 787-794. 8 N. Akhtar and S. Mullick, Energy, 2007, 32, 1067-1074.

9 N. Akhtar and S. C. Mullick, Int. J. Heat Mass Transfer, 2012, 55, 125-132.

10 Q. Z. Huang, J. F. Shi, L. L. Wang, Y. J. Li, L. W. Zhong and G. Xu, Thin Solid Films, 2016, 610, 19-25.

11 A. A. Hegazy, Renewable Energy, 2001, 22, 525-540.

12 M. C. Bautista and A. Morales, Sol. Energy Mater. Sol. Cells, 2003, 80, 217-225.

13 G. San Vicente, A. Morales, N. Germán, S. Suarez and B. Sánchez, J. Sol. Energy Eng., 2012, 134, 041011.

14 Z. Hua-Yu, C. Jian-Jun, L. I. Dong-Shuang, Z. Wei-Li, F. U. Dong-Ju, M. A. Qing, W. Xiao-Wei, Z. Li-Jie and T. A. N. Man-Lin, J. Inorg. Mater., 2016, 31, 365.

15 B. N. Sahoo and B. Kandasubramanian, $R S C A d v .$, 2014, 4, 22053.

16 S. Nishimoto and B. Bhushan, RSC Adv., 2013, 3, 671-690. 17 S. H. Kim, J. Adhes. Sci. Technol., 2008, 22, 235-250.

18 N. Cohen, A. Dotan, H. Dodiuk and S. Kenig, Mater. Manuf. Processes, 2015, 31, 1143-1155.
19 Y. Si and Z. Guo, Nanoscale, 2015, 7, 5922-5946.

20 D. Su, C. Huang, Y. Hu, Q. Jiang, L. Zhang and Y. Zhu, Appl. Surf. Sci., 2011, 258, 928-934.

21 Y. Zhu, J. Shi, Q. Huang, L. Wang and G. Xu, Chem. Commun., 2017, 53, 2363-2366.

22 G. Liu, W. S. Y. Wong, N. Nasiri and A. Tricoli, Nanoscale, 2016, 8, 6085-6093.

23 Q. Xu, Q. Zhao, X. Zhu, L. Cheng, S. Bai, Z. Wang, L. Meng and Y. Qin, Nanoscale, 2016, 8, 17747-17751.

24 L. H. Joo and M. Stephen, J. Polym. Sci., Part B: Polym. Phys., 2010, 45, 253-261.

25 Y. Y. Yan, N. Gao and W. Barthlott, Adv. Colloid Interface Sci., 2011, 169, 80-105.

26 J. Gao, J. S.-P. Wong, M. Hu, W. Li and R. K. Y. Li, Nanoscale, 2014, 6, 1056-1063.

27 J. Zarzycki, J. Sol-Gel Sci. Technol., 1997, 8, 17-22.

28 Y. Sun, X. Xiao, G. Chai, G. Xu, B. Xiong and S. Zhang, Mater. Express, 2014, 4, 205-212.

29 J. Shi, Y. Sun, Q. Huang, Y. Zhu, L. Wang, L. Zhong and G. Xu, Mater. Express, 2016, 6, 301-309. 